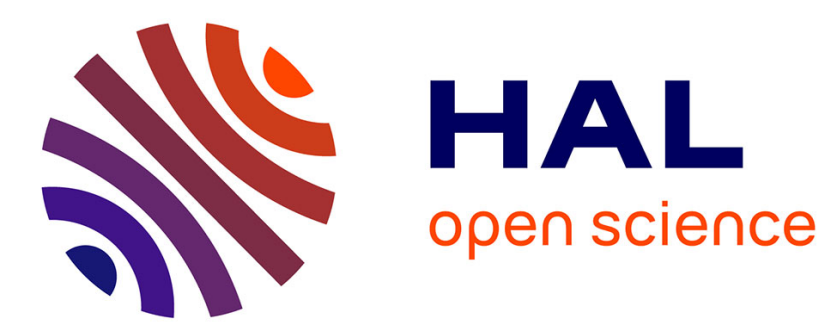

\title{
Nonlocal acoustic metasurface for ultrabroadband sound absorption
}

Yifan Zhu, Aurélien Merkel, Krupali Donda, Shiwang Fan, Liyun Cao, B.

Assouar

\section{> To cite this version:}

Yifan Zhu, Aurélien Merkel, Krupali Donda, Shiwang Fan, Liyun Cao, et al.. Nonlocal acoustic metasurface for ultrabroadband sound absorption. Physical Review B, 2021, 103 (6), pp.064102. 10.1103/PhysRevB.103.064102 . hal-03412412

\section{HAL Id: hal-03412412 \\ https://hal.science/hal-03412412}

Submitted on 3 Nov 2021

HAL is a multi-disciplinary open access archive for the deposit and dissemination of scientific research documents, whether they are published or not. The documents may come from teaching and research institutions in France or abroad, or from public or private research centers.
L'archive ouverte pluridisciplinaire HAL, est destinée au dépôt et à la diffusion de documents scientifiques de niveau recherche, publiés ou non, émanant des établissements d'enseignement et de recherche français ou étrangers, des laboratoires publics ou privés. 


\title{
Nonlocal acoustic metasurface for ultra-broadband sound
}

\author{
absorption \\ Yifan Zhu*, Aurélien Merkel, Krupali Donda, Shiwang Fan, Liyun Cao, and Badreddine \\ Assouar* \\ Institut Jean Lamour, CNRS, Université de Lorraine, 54000 Nancy, France.
}

\begin{abstract}
Classical meta-absorber designs usually have a trade-off between bandwidth, efficiency and thickness. Here, we introduce the concept of nonlocal acoustic metasurface absorber by using a bridge structure connecting resonating unit cells to improve the performances of the meta-absorber. By utilizing the coupling effect between the adjacent unit cells, ultra-broadband sound absorption is achieved with deep-wavelength thickness. The physical mechanism of the nonlocal acoustic metasurface absorber is investigated and uncovered by developing analytical models. We theoretically and numerically study the nonlocal metasurface with connecting bridge and the traditional metasurface without bridge. The nonlocality can introduce three specific effects; the optimization of effective acoustic impedances, the shift of Fabry-Perot resonant frequencies, and the enhancement of the coupling effects between adjacent unit cells. These effects contribute to improve the bandwidth and the efficiency of the acoustic meta-absorber. We numerically and experimentally achieve an average absorption coefficient larger than 0.9 within the ultrabroadband bandwidth extending from about $600 \mathrm{~Hz}$ to $2600 \mathrm{~Hz}$, with a metasurface of $6.8 \mathrm{~cm}$, viz., $\lambda / 9$ for the lowest frequency. Our finding demonstrates the advantage of non-local acoustic metasurface to conceive subwavelength sound meta-absorber.
\end{abstract}

Keywords: Nonlocal acoustic metasurfaces, Sound absorption, Acoustic metamaterials.

\section{Corresponding authors}

*yifan.zhu@univ-lorraine.fr

*badreddine.assouar@univ-lorraine.fr 


\section{Introduction}

Conventional acoustic metasurfaces have the ability to manipulate acoustic wavefront based on generalized Snell's law and the phase discontinuities between adjacent unit cells. The phase shifts at the interface of metasurface can lead to various wavefront manipulations, such as extraordinary refraction/reflection, acoustic focusing, non-diffraction beams, sound absorption, and so on [1]. In the past several years, acoustic metasurfaces have been employed to design sound absorbers [2-12] with optimized performances, which is difficult to be realized by traditional sound absorbing materials. For example, the thickness of acoustic metasurface absorber can be largely decreased to deep subwavelength dimension with highly resonant unit cells [2-6], especially for low-frequency regime. Furthermore, the bandwidth of acoustic absorber can be improved via combination of multiple unit cells with different resonant frequencies, to achieve broadband sound meta-absorber [7-12]. The design of absorbing metasurface is actually dictated by the trade-off between bandwidth, efficiency and thickness.

On the other hand, the concept of nonlocality has been widely studied in both optics [13] and optical metasurface [14-15]. Nonlocality can be seen as a more exact theoretical description of the effective medium, that considers nonlocal responses from transverse coupling. Drawing inspiration from their optical counterparts, recently, the mechanism of nonlocal metasurface has been extended to acoustics [16-19], which take advantages of the nonlocal response due to the transverse coupling between adjacent acoustic metastructure unit cells. This physical mechanism is completely different from the one on which are based conventional acoustic metasurfaces. For example, the latter can hardly realize perfect extraordinary refraction/reflection when the incident angle is large, due to the fact that the high-order diffractions could influence the acoustic field distributions near the surface of the metasurfaces. The concept of nonlocal acoustic metasurface 
[16] can overcome this problem. For instance, optimized meta-grating structures based on nonlocality have been designed to realize high-efficient extraordinary reflection [16] refraction [17] and beam splitting [18]. The nonlocal metasurface is further designed to realize hyperbolic sound propagation [19], via connection between the adjacent unit cells of the metasurface, showing the unique advantages of the nonlocal acoustic metasurfaces.

In this research, we propose the concept of nonlocal metasurfaces to conceive a sound absorber, showing the interest of nonlocal couplings which interestingly increase their efficiency. We will investigate and analyze our concept based on theoretical, numerical and experimental approaches, and discuss in details the involved physical mechanism.

\section{Model of nonlocal metasurface and theory}

We first compare two conventional designs of acoustic meta-absorbers with multiple unit cells based on Fabry-Perot (FP) resonances as schematically shown in Fig. 1(a) and 1(b), whose mechanism are similar to previous two designs in Refs. 7 and 11, respectively. The supercell of the first one consists of 22 straight channels with high filling ratio at the surface [7], and large height under the surface. Then, in order to gain space, the supercell of the second one consists of 22 folded channels with low filling ratio [11] at the surface, and smaller height under it. The comparison between the two designs indicates that there is always a trade-off between the absorption efficiency and the structure thickness, since the second structure is less efficient than the first one in term of absorption. When the surface filling ratio change from 0.7 to 0.2 , the structure thickness is decreased from $14.6 \mathrm{~cm}$ to $6.6 \mathrm{~cm}$, but the averaged absorption efficiency is decreased from 0.92 to 0.56 . Based on the previous theory [7], the absorption efficiency is directly determined by the filling ratio. Here comes the question: Can we get a high absorption with a low surface filling ratio 
in the meta-absorber?

Indeed, we will show that if we introduce the nonlocality effect in the second design, we can improve the absorption efficiency and bandwidth, even with a very low surface filling ratio. In order to introduce the nonlocal effect, a bridge structure is deliberately designed to connect the unit cells, as a key element to make the supercell totally continuous. Therefore, the efficiency and bandwidth can be improved. We compare three particular cases, that is, the original folded metasurface without bridge, the metasurface with cavities attached on the channel, and the metasurface with optimized bridge attached on the folded channels to connect all the unit cells, respectively. The acoustic metasurface with optimized bridge is defined as a nonlocal metasurface. By comparing nonlocal acoustic metasurface with the local one, the mechanism of nonlocal acoustic metasurface absorber (NAMA) is investigated and revealed via developing and analyzing effective circuit models. The coupled resonances between the unit cells lead to high efficiency and continuous frequency band. We explore the advantage of nonlocal acoustic metasurface to conceive subwavelength sound absorber, which could have practical application for noise control, environmental acoustics, and so on.

Two classical acoustic absorbing metasurfaces based on multiple FP resonant units are shown in Figs. 1(a) and 1(b). The supercell is marked by red dashed rectangle with a surface size of 4.5 $\mathrm{cm} \times 1.5 \mathrm{~cm}(3 w \times w)$. Both structures shown in Figs. 1(a) and 1(b) contain three supercells. The first classical design shown in Fig. 1(a) contains 22 straight channels with channel lengths $L(n)$ ranging from $4.7 \mathrm{~cm}$ to $14.6 \mathrm{~cm}$ as the black curve shown in Fig. 1(c). The width of the channels is $w 0=1.4$ $\mathrm{cm}$. The thickness of the channels is $t_{1}=0.15 \mathrm{~cm}$, about 20 to 30 times of the thickness of thermalviscous boundary layer [11]. The second one shown in Fig. 1(b) contains 22 folded channels with same effective channel lengths. The folding feature makes the all the folded channels have limited 
heights $(H(n)<6.6 \mathrm{~cm})$. The width of the fold channels is $w_{1}=0.4 \mathrm{~cm}$. Since the channels with different effective lengths correspond to different resonant absorption frequencies, this type of meta-absorber can realize broadband sound absorption. If we considering a Lorentzian form of resonance and all order resonance modes, the effective acoustic impedance of the meta-absorber can be expressed as $[2,7,20]$ :

$$
Z=\frac{i \pi Z_{0}}{4 \omega \gamma}\left(\sum_{n=1}^{22} \sum_{m=0}^{\infty} \frac{\Omega_{m n}}{\left(\Omega_{m n}^{2}-\omega^{2}-i \beta \omega\right)}\right)^{-1} \equiv\left(\sum_{n=1}^{22} \frac{1}{Z(n)}\right)^{-1}
$$

where $Z_{0}=\rho_{0} c_{0}$ is the air impedance, $\rho_{0}=1.21 \mathrm{~kg} / \mathrm{m}^{3}$ is air density and $c_{0}=343 \mathrm{~m} / \mathrm{s}$ is the sound speed in the air, $\omega=2 \pi f$ is the angular frequency, $\Omega_{m n}=2 \pi f(m, n)$ denotes the $m$-order resonant angular frequency of $n$th straight/folded channel, $\beta$ is the dissipation coefficients of the acoustic metastructure, and $Z(n)$ is the acoustic impedance of $n$th channel. The filling ratio $\gamma$ is defined as the ratio of the surface area occupied by channels' cross sections to the effective surface area exposed to sounds. The total acoustic impedance of the supercell is defined as $Z \equiv p / v, p$ being the sound pressure modulation and $v$ the displacement velocity in response to the pressure at the surface of the supercell.

The absorption coefficient is calculated by:

$$
A=1-\left|\frac{Z-Z_{0}}{Z+Z_{0}}\right|^{2}
$$

For the ideal case, when the channels number is large enough and their eigenfrequencies are gradient-varying, the impedance and the absorption can be approximated as [7]:

$$
\begin{gathered}
\frac{Z}{Z_{0}}=\frac{\pi}{\gamma\left[\pi-2 i \tanh ^{-1}\left(\Omega_{1} / \omega\right)\right]} \\
A=1-\left|\frac{(1-\gamma) \pi+2 i \gamma \tanh ^{-1}\left(\Omega_{1} / \omega\right)}{(1+\gamma) \pi-2 i \gamma \tanh ^{-1}\left(\Omega_{1} / \omega\right)}\right|^{2}
\end{gathered}
$$


Here, $\Omega_{11}=2 \pi f(1,1)$ is the cutoff (lowest) resonant angular frequency of straight/folded channel. The absorption calculated by Eq. (4) for the ideal case is plotted by dashed lines in Fig. 1(d). The averaged absorption within $600 \mathrm{~Hz}$ to $2600 \mathrm{~Hz}$ for the two structures with $\gamma_{0}=0.7$ and $\gamma_{1}=0.2$ are $A_{\text {ideal } 0}=0.97$, and $A_{\text {ideal }}=0.56$, respectively, as shown in Eq. 4 . The absorption depends directly on the surface filling ratio, the low $\gamma$ value will induce impedance mismatch at the interface. The approximated relation can be given as:

$$
A \approx \frac{4 \gamma}{(1+\gamma)^{2}}
$$

The simulated absorptions for the two structures are plotted by solid lines in Fig. 2(d). Throughout the paper, the numerical simulations are accomplished by COMSOL Multiphysics 5.5a. The averaged simulated absorptions for the two structures within $600 \mathrm{~Hz}$ to $2600 \mathrm{~Hz}$ are $A_{0}=0.92$, and $A_{1}=0.56$, respectively, which agree well with the analytical approach. A perfectly flat absorption requires an infinite number of resonance modes, which is not possible in a real system. Thus, the absorption spectrum of our systems with a finite number of resonance modes are not perfectly flat. In the following, we will introduce the nonlocal effect in the second design with folded channel to achieve a NAMA. The design goal is to have a high efficient absorption even with a low filling ratio $\gamma_{1}=0.2$, as well as a continuous bandwidth with an optimized profile. 
(a)

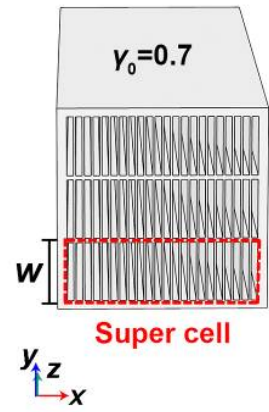

(b)

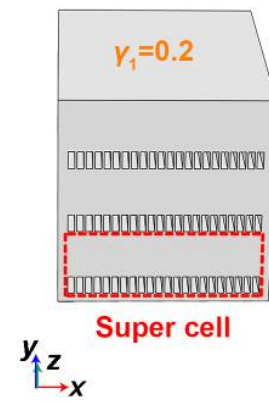

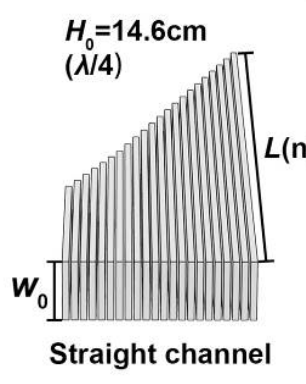

(c)

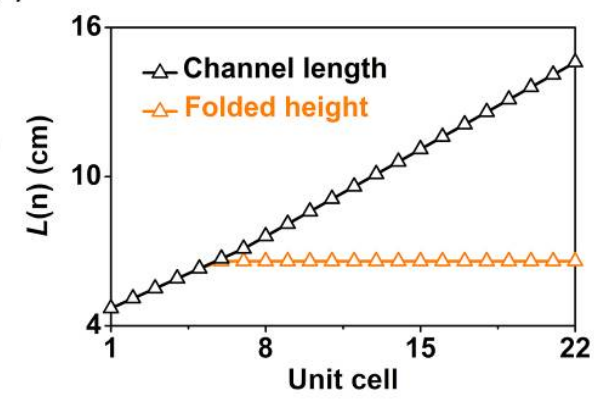

(d)

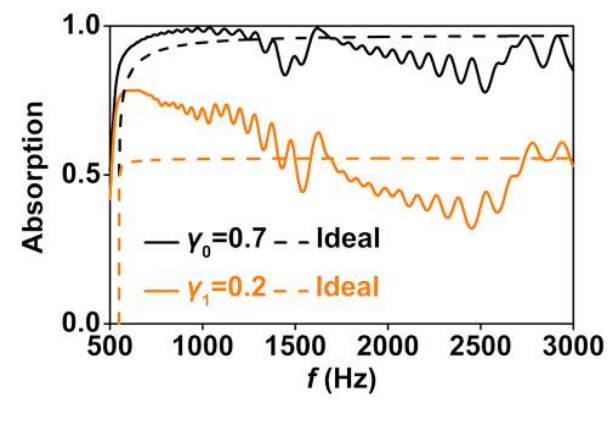

FIG. 1. The comparison of two classical meta-absorbers. (a) The structure of the first metaabsorber whose supercell consists of 22 straight channels with surface filling ratio of $\gamma_{0}=0.7$ and height of $H_{0}=14.6 \mathrm{~cm}$. (b) The structure of the second meta-absorber whose supercell consists of 22 folded channels with surface filling ratio of $\gamma_{1}=0.2$ and height of $H_{1}=6.6 \mathrm{~cm}$. (c) The channel lengths $(4.7 \mathrm{~cm}$ to $14.6 \mathrm{~cm})$ and the folded channel heights $(4.7 \mathrm{~cm}$ to $6.6 \mathrm{~cm})$ for 22 units. (d) The simulated (solid lines) and ideal (dashed lines) absorption coefficient for the first and second metaabsorber. The averages of the simulated absorption within the range of $600 \mathrm{~Hz}$ to $2000 \mathrm{~Hz}$ are $A_{0}=0.92$, and $A_{1}=0.56$, while the ideal absorptions from Eq. (4) are $A_{\text {ideal0 }}=0.97$, and $A_{\text {ideal } 1}=0.56$ for the first and second meta-absorber, respectively.

In order to reduce the impedance mismatch of the folded meta-absorber shown Fig. 2(a), we design an array of cavities to be attached on the folded channels, as shown in Fig. 2(b). The height of cavities is $h_{1}=1.2 \mathrm{~cm}$, and width is $w_{2}=1 \mathrm{~cm}$. The two absorbers shown in Figs. 2(a-b) are both 
traditional local acoustic metasurface, with absence of connections between adjacent unit cells. A nonlocal metasurface requires obvious coupling between adjacent unit cells [19]. In order to introduce the nonlocal effect in the metasurface absorber, a coupling bridge structure is designed to be attached on the channel array, which connects all the channels as shown in Fig. 2(c). The height of the optimized bridge is gradient varying from $h_{2}=1.2 \mathrm{~cm}$ to $h_{3}=2.4 \mathrm{~cm}$ $(h(x)=0.012+0.267 x)$. The fact that the different channels are connected behind the surface of the metasurface results in a nonlocal surface impedance. The metasurface with internal bridge shown in Fig. 3(c) is defined as a nonlocal acoustic metasurface.

In order to analytically analyze the above different metasurfaces, effective circuit models are built for the three models: without bridge (O), with cavity (C), and with bridge (B) as illustrated in Figs. 2(d-f), respectively. The acoustic impedance of $n^{\text {th }}$ branch consists of two parts, that is, the acoustic impedances $Z_{\mathrm{O}}(n), Z_{\mathrm{C}}(n), Z_{\mathrm{B}}(n)$, of $n^{\text {th }}$ unit cell, and the acoustic impedances $Z_{\mathrm{OM}}(n)$, $Z_{\mathrm{CM}}(n), Z_{\mathrm{BM}}(n)$, induced by the transverse coupling of the neighboring unit cells.

For the $\mathrm{O} / \mathrm{C} / \mathrm{B}$ cases, the acoustic impedance of the $n$th unit cell shown in Fig. 2(d-f) can be written with the same form of Eq. (1) with $Z_{\mathrm{O} / \mathrm{C} / \mathrm{B}}(n)=Z(n)$, expanded as:

$$
Z_{\mathrm{O} / \mathrm{C} / \mathrm{B}}(n)=\frac{i \pi Z_{0}}{4 \omega \gamma_{\mathrm{O} / \mathrm{C} / \mathrm{B}}}\left(\sum_{m=0}^{\infty} \frac{2 \pi f_{\mathrm{O} / \mathrm{C} / \mathrm{B}}(m, n)}{\left.\left[2 \pi f_{\mathrm{O} / \mathrm{C} / \mathrm{B}}(m, n)\right]^{2}-\omega^{2}-i \beta \omega\right)}\right)^{-1}
$$

Then, the total impedance of an effective circuit can be expressed as:

$$
Z_{\mathrm{O} / \mathrm{C} / \mathrm{B}}=\left(\sum_{n=1}^{22} \frac{1}{Z_{\mathrm{O} / \mathrm{C} / \mathrm{B}}(n)+Z_{\mathrm{OM} / \mathrm{CM} / \mathrm{BM}}(n)}\right)^{-1}
$$

In the following, we will show the different mechanisms for the physical quantities of $\gamma_{0 / C / B}$,

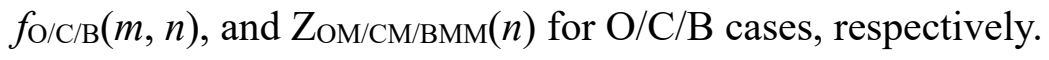

The first effect of the inserted cavity or bridge is the improvement of the acoustic impedance matching, which can be also regarded as the increasing of the effective filling ratio, although the 
original filling ratio for $\mathrm{O} / \mathrm{C} / \mathrm{B}$ cases are all 0.2 . For the $\mathrm{O}$ case, the unit cell is a folded channel without any attachment. The filling ratio is original $\gamma_{0}=S_{1} / S=\left(t_{1} \times w_{1}\right) /(t \times w)=0.2$. In this case, the predicted ideal absorption by Eq. (5) is only $A_{\text {idealo }}=0.56$. For the $\mathrm{C}$ case, the inserted cavities can improve the acoustic impedance matching between the unit cells and the free space. We characterize it as a change of effective filling ratio, which is related to the volume filling ratio of the cavity, expressed as

$$
\gamma_{\mathrm{C}} \approx \frac{t_{1} w_{1} H+t_{1} w_{2} h_{1}}{t w H},
$$

where $H=2.8 \mathrm{~cm}$ is the maximal height of the cavity or bridge, as marked in Fig. 2(g). We get the value of $\gamma_{\mathrm{C}}=0.41$. The ideal absorption is $A_{\text {idealC }}=0.82$ deduced by Eq. (5). For the B case, the effective filling ratio is also related to the volume filling ratio, calculated as:

$$
\gamma_{B} \approx \frac{t_{1} w_{1} H+\int_{0}^{3 w} t_{1} w_{2} h(x) d x}{t w H},
$$

Since the bridge is designed with a shape of $h(x)=0.012+0.267 x$, we get the value of $\gamma_{\mathrm{B}}=0.61$. Thus, the predicted ideal absorption is increased to $A_{\text {idealB }}=0.93$.

The second effect of the inserted cavity or bridge is the shifts of the FP resonant frequency. For an original channel array for the $\mathrm{O}$ case, the $m^{\text {th }}$ order resonant frequency for $n^{\text {th }}$ unit cell is [21]

$$
f_{\mathrm{O}}(m, n)=\frac{(2 m-1) c_{0}}{4 L(n)},
$$

where $L(n)$ comprises the length of the channel [Fig. 1(c)] and an additional length induced by the radiation at the opening [22]. However, for the $\mathrm{C}$ case, the insert cavity influences the original resonant frequency, the $m^{\text {th }}$ order resonant frequency for $n^{\text {th }}$ unit cell becomes: 


$$
f_{\mathrm{C}}(m, n)=\frac{(2 m-1) c_{0}}{4 L(n)-h_{1}},
$$

in which the height of the cavities is considered to decrease the effective length of the channel. For the $\mathrm{B}$ case, the $m^{\text {th }}$ order resonant frequency for $n^{\text {th }}$ unit cell becomes:

$$
f_{\mathrm{B}}(m, n)=\frac{(2 m-1) c_{0}}{4 L(n)-4 h(n)},
$$

in which the local height of the bridge is considered to apparently decrease the effective length of the channel.

The third effect is that the inserted bridge can strengthen the transverse coupling between neighboring channels. For the $\mathrm{O} / \mathrm{C} / \mathrm{B}$ cases, the acoustic impedance induced by the coupling effects at the opening of the channel is expressed as [23-24]:

$$
Z_{\text {OM/CM/BM } 0}(n)=\frac{Z_{\mathrm{M}(n-1)(n)}{ }^{2}}{Z_{\text {O//B }}(n-1)}+\frac{Z_{\mathrm{M}(n)(n+1)}{ }^{2}}{Z_{\mathrm{O} / \mathrm{C} / \mathrm{B}}(n+1)},
$$

where $Z_{\mathrm{M}(n-1)(n)}$ is the mutual impedance between $(n-1)^{\text {th }}$ and $n^{\text {th }}$ unit cells. The mutual impedance between the unit cells is defined as the average sound pressure on one of the channel openings, generated by a unit volume velocity on the other [23]. The schematic diagram for calculation of the mutual impedance is shown in Fig. 2(g). The mutual impedance $\mathrm{Z}_{\mathrm{M}(n-1)(n)}$ is calculated as:

$$
Z_{M(n-1)(n)}=\frac{i \omega \rho_{0}}{4 \pi} \frac{1}{t_{1}} \iint_{s} \frac{e^{-i k \chi}}{\chi} d S=\frac{i \omega \rho_{0}}{2} \frac{1}{t_{1}} \int_{-t_{1} / 2}^{t_{1} / 2} \frac{e^{-i k \sqrt{a_{a c}{ }^{2}+(t-\xi)^{2}}}}{\sqrt{l_{a c}^{2}+(t-\xi)^{2}}} d \xi,
$$

where $l_{\mathrm{ac}} \approx 0.425 t_{1}$ is the effective distance from the opening of the channel to the assumed monopole sound source [23]. $t_{1}=0.15 \mathrm{~cm}$ is size of the channel in $x$-direction, $t=0.2 \mathrm{~cm}$ is the distance between two neighboring channels.

As shown in Fig. 2(g), the coupling effects for the $\mathrm{O}$ and $\mathrm{C}$ cases only exist at the openning of the channel, while for the B case, the coupling effects exist at not only the opening of the channel, 
but also inside the bridge. Therefore, we should consider the mutual impedance twice with a two times iterative calcultion. When the mutual impedance is a first order small quantity of the acoutsic impedance of unit cell, the final total mutual impedance is $Z_{\mathrm{BM}}(n) \approx 2 Z_{\mathrm{BM} 0}(n)$. This additional mutual impedance contributes to the nonlocal acoustic impedance for the B case, which can improve the final sound abosorption spectrum of a nonlocal meatsurface. The bridge shape has been optimized by simulations for obtaining a desired abosorption spectrum, and we finally choose the one with the gradient heights of $h(x)=0.012+0.267 x$.

(a)

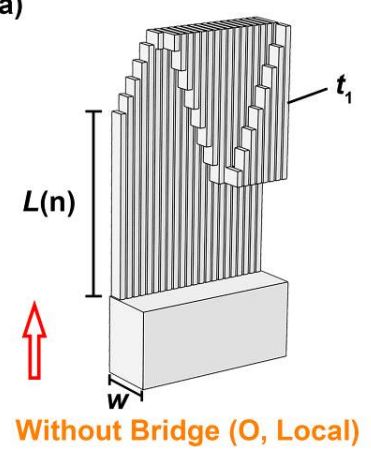

(d)

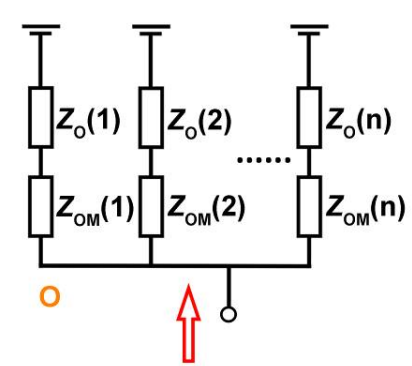

(b)

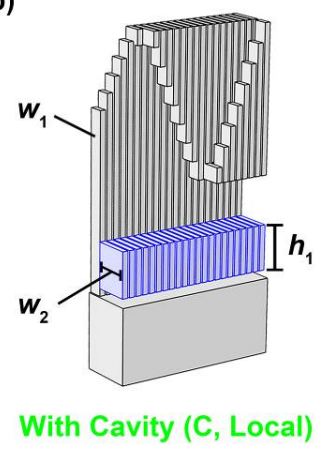

(e) (c)

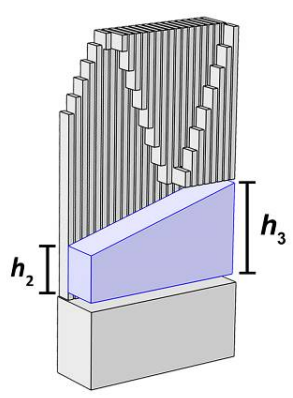

With Bridge (B, Nonlocal)

(f)

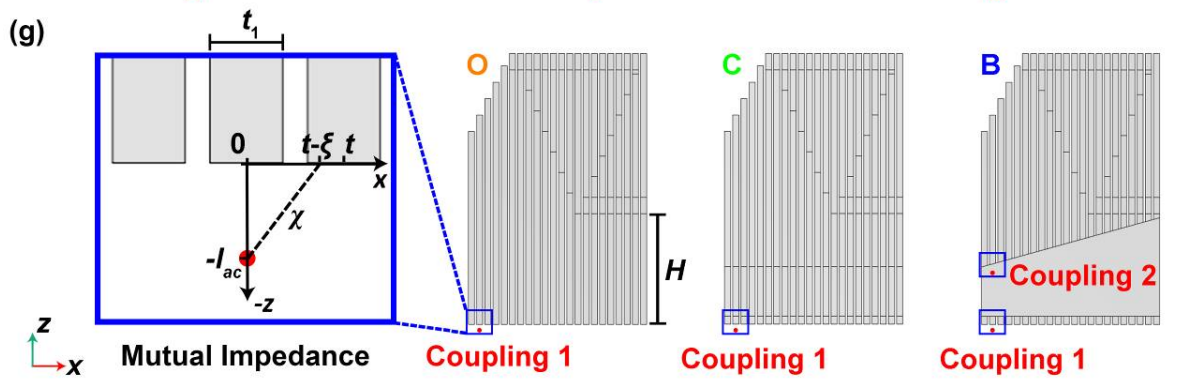

FIG. 2. The structures of the supercell (a) without bridge (O, local metasurface), (b) with cavity (C, local metasurface), and (c) with bridge (B, nonlocal metasurface), respectively. The structural 
parameters are marked in the figure. The effective circuit models for the cases of (d) O (e) C (f) B, respectively. (g) The schematic diagram of the transverse coupling effect between adjacent unit cells.

\section{Numerical simulation and analysis}

We, afterwards, have numerically investigated the performance of the $\mathrm{O} / \mathrm{C} / \mathrm{B}$ structures. In COMSOL simulations, the multiphysics module of "Acoustic-Thermovisous Acoustic Interaction, Frequency Domain" is used. The regions of the folded channels are built in "Thermovisous Acoustics, Frequency Domain" module. The regions of cavities, bridges and the outside air are built in "Pressure Acoustics, Frequency Domain" module. The boundaries of the folded channel are set as sound hard boundaries. Boundary layers meshes are built in the channel for the acoustic absorption simulation.

Figure 3(a) shows all the existing resonant modes within the range of [500 Hz, $3000 \mathrm{~Hz}]$ for the $\mathrm{O} / \mathrm{C} / \mathrm{B}$ cases. The simulated 1-order resonant modes for these cases are within the range of [520 Hz, $1590 \mathrm{~Hz}],[540 \mathrm{~Hz}, 1650 \mathrm{~Hz}]$, and [630 Hz, $2140 \mathrm{~Hz}]$, respectively, which agree with the analytical results calculated by Eqs. (10)-(12) [marked by circles in Fig. 1(a)]. The simulated 2order resonant modes for the $\mathrm{O} / \mathrm{C} / \mathrm{B}$ cases are within $[1640 \mathrm{~Hz}, 3000 \mathrm{~Hz}],[1670 \mathrm{~Hz}, 3000 \mathrm{~Hz}]$, and $[1960 \mathrm{~Hz}, 3000 \mathrm{~Hz}]$, respectively. It is noted that two small frequency gaps between 1-order and 2-order modes are observed for $\mathrm{O}$ and $\mathrm{C}$ cases, corresponding to the range of [1590 Hz, 1640 $\mathrm{Hz}]$ and $[1650 \mathrm{~Hz}, 1670 \mathrm{~Hz}]$, respectively. Similarly, a small overlapping frequency region between 1-order and 2-order modes is observed for the B case within [1960 Hz, $2140 \mathrm{~Hz}]$. The simulated 3-order resonant modes for the $\mathrm{O}$ and $\mathrm{C}$ cases are within $[2750 \mathrm{~Hz}, 3000 \mathrm{~Hz}]$ and [2580 $\mathrm{Hz}, 3000 \mathrm{~Hz}]$, respectively, while the 3 -order resonant modes for the B case is higher than 3000 
$\mathrm{Hz}$, not shown in the figure.

Figure 3(b) exhibits the numerical sound absorption coefficients within $500 \mathrm{~Hz}$ to $3000 \mathrm{~Hz}$ frequency range for the cases $\mathrm{O} / \mathrm{C} / \mathrm{B}$, respectively. The average absorption coefficient for the $\mathrm{O}$ case is only 0.56 (orange curve) within the range of [600 Hz, $2600 \mathrm{~Hz}$, same as the result in Fig. 1(d). The average absorption coefficient for the $\mathrm{C}$ case is 0.78 (green curve) within [600 Hz, 2600 Hz]. It increases from 0.56 to 0.78 due to the modulation of the acoustic impedance of each channel by the cavities. The average absorption coefficient for the B case within $[600 \mathrm{~Hz}, 2600 \mathrm{~Hz}]$ is 0.9 , which is obviously larger than the cases of $\mathrm{O}$ and $\mathrm{C}$ of the local metasurface. In addition, we have the absorption coefficient $A>0.8$ within [625 Hz, $2545 \mathrm{~Hz}$. Considering a wall thickness of 0.2 $\mathrm{cm}$, the total thickness of the NAMA is $6.8 \mathrm{~cm}$, that is $\lambda / 9$ of the lowest working frequency.

We have then analyzed different modes within the curves in Figs. 3(a) and 3(b). The modes of O1 $(1000 \mathrm{~Hz}), \mathrm{C} 1(1060 \mathrm{~Hz})$, and B1 $(1280 \mathrm{~Hz})$ are corresponding to 1-order resonances at $8^{\text {th }}$ unit cells. The acoustic pressure distributions for $\mathrm{O} 1, \mathrm{C} 1, \mathrm{~B} 1$ are shown in Fig. 3(c). The acoustic pressure amplitude for O1, C1, B1 are 28.3, 32.2, and 35.3, respectively. The corresponding sound absorptions in Fig. 3(b) for O1, C1, B1 are $0.72,0.80$ and 0.89 , respectively. These results suggest that the B case has the highest sound intensity inside the FP channel, which lead to the highest sound absorption.

The modes of O2 $(1580 \mathrm{~Hz}), \mathrm{C} 2(1580 \mathrm{~Hz})$, and B2 $(1580 \mathrm{~Hz})$ are located at an interesting frequency close to the mode gaps for $\mathrm{O}[1590 \mathrm{~Hz}, 1640 \mathrm{~Hz}]$ and $\mathrm{C}[1650 \mathrm{~Hz}, 1670 \mathrm{~Hz}]$ case. Figure 3(b) shows that two absorption valleys are observed at $1580 \mathrm{~Hz}$ for the $\mathrm{O}$ and $\mathrm{C}$ cases with low absorption of 0.54 and 0.68 [marked by Abnormal 1 in Fig. 3(b)], while no valley is observed for the $\mathrm{B}$ case at $1580 \mathrm{~Hz}$ with the absorption of 0.90 . The acoustic field distributions in the supercells are shown in Fig. 3(d). Observing the acoustic field distributions of O1, C1, B1 and B2, the strong 
resonance of $n^{\text {th }}$ channel can also induce weak resonance in $(n-1)^{\text {th }}$ and $(n+1)^{\text {th }}$ channel. This is owing to the Lorentzian form of the resonance of FP channel that has a small bandwidth. Thus, for the $\mathrm{O} 2$ and $\mathrm{C} 2$ cases, the incident frequency can induce the resonances in $22^{\text {nd }}, 1^{\text {st }}$ and $2^{\text {nd }}$ channels, while there is no transverse coupling between $22^{\text {nd }}$ and $1^{\text {st }}$ channels since they are geometrically separated. Therefore, the inherent decoupling between $22^{\text {nd }}$ and $1^{\text {st }}$ channels, and the small frequency gaps of $[1590 \mathrm{~Hz}, 1640 \mathrm{~Hz}]$ and $[1650 \mathrm{~Hz}, 1670 \mathrm{~Hz}]$ lead to the absorption valley in the $\mathrm{O}$ and $\mathrm{C}$ cases. While for the $\mathrm{B}$ curve, there is no apparent absorption valley, because the small frequency overlapping region $[1960 \mathrm{~Hz}, 2140 \mathrm{~Hz}]$ compensates the decoupling effect between $22^{\text {nd }}$ and $1^{\text {st }}$ channels.

The modes of O3 $(1980 \mathrm{~Hz}), \mathrm{C} 3(2005 \mathrm{~Hz})$, and B3 $(2395 \mathrm{~Hz})$ correspond to 2-order resonances at $17^{\text {th }}$ unit cells. The acoustic pressure distributions for O2, C2, B2 are shown in Fig. 3(e). The acoustic pressure amplitude of O3, C3, B3 are 14.8, 19.6, and 22.9, respectively. The corresponding sound absorptions showed in Fig. 3(b) are 0.46, 0.79 and 0.95, respectively. These results suggest that the 2-order mode for B case has the highest sound intensity inside the FP channel, similar to 1-order mode, which lead to the highest sound absorption.

The modes of $\mathrm{O} 4(2880 \mathrm{~Hz}), \mathrm{C} 4(2880 \mathrm{~Hz})$, and $\mathrm{B} 4(2880 \mathrm{~Hz})$ are located at an interesting frequency at high frequency region. The acoustic field distributions are shown in Fig. 3(f). O4 and C4 modes can induce 2-order and 3-order modes in the supercells, while B4 mode can only induce 2-order mode, which agree with the mode distributions in Fig. 3(a). An abnormal phenomenon [marked by Abnormal 2 in Fig. 3(a)] is observed indicating that the analytical solution for the C case for 3 -order mode does not agree with the simulated one. This is due to the fact that standing wave is excited between adjacent unit cells [25] for C4 case as shown in Fig. 3(f). Another abnormal phenomenon [marked by Abnormal 3 in Fig. 3(b)] indicates that at $2880 \mathrm{~Hz}$, the 
absorptions for $\mathrm{O} 4, \mathrm{C} 4$, and $\mathrm{B} 4$ cases are $0.55,0.99,0.36$, respectively. The $\mathrm{C}$ case has the highest absorption, much larger than the $\mathrm{O}$ and $\mathrm{B}$ ones. This phenomenon is different from the range of [600 Hz, $2600 \mathrm{~Hz}]$, which is due to the fact that the standing wave in the resonators can significantly increase the sound absorption [4, 26], and the mode density at C4 (consists of both 2-order and 3-order modes) is higher than B4. The absorption coefficient, sound pressure amplitude in the resonant channels, and sound pressure amplitude in their adjacent channels for all interested modes are shown in Fig. 3(g). The results suggest that the sound absorption usually increases with the sound pressure amplitude in the resonant channels and their adjacent ones. 


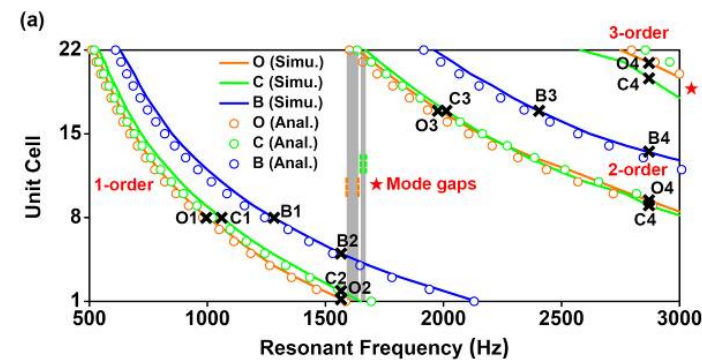

(b)

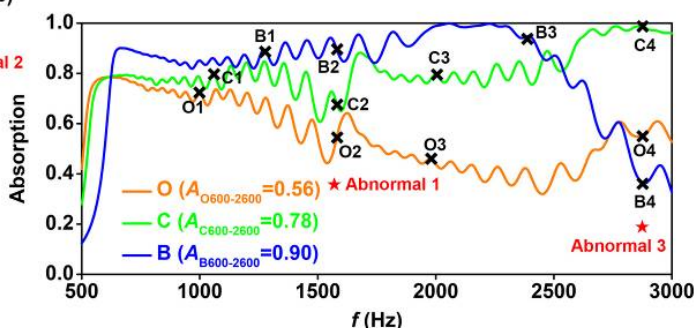

(c)
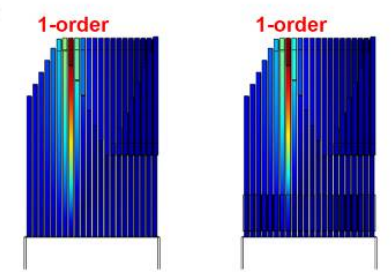

$01: \mathbf{1 0 0 0 \mathrm { Hz }}$
$\boldsymbol{A}_{01}=\mathbf{0 . 7 2 ,} \boldsymbol{P}_{01}=\mathbf{2 8 . 3}$

$\mathrm{C} 1: 1060 \mathrm{~Hz}$

$A_{\mathrm{c} 1}=0.80, P_{\mathrm{c} 1}=32.2$

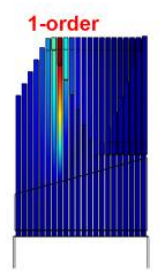

B1: $1280 \mathrm{~Hz}$

$A_{\mathrm{B} 1}=0.89, P_{\mathrm{B} 1}=35.3$
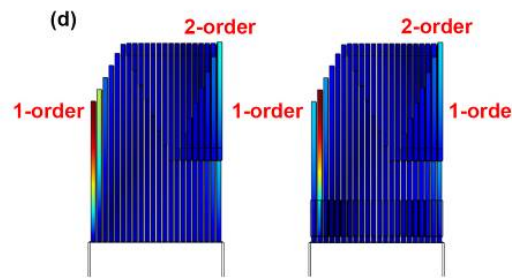

$\mathrm{O} 2: 1580 \mathrm{~Hz}$ $A_{\mathrm{O} 2}=0.54, P_{\mathrm{o} 2}=26.0$

$\mathrm{C} 2: 1580 \mathrm{~Hz}$ $A_{\mathrm{c} 2}=0.68, P_{\mathrm{c} 2}=31.9$

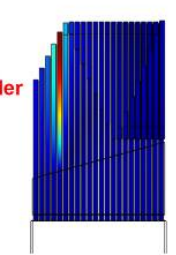

(e)
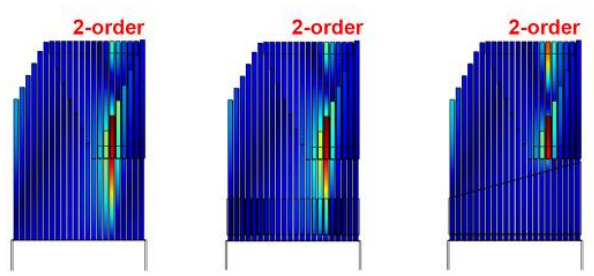

(f)
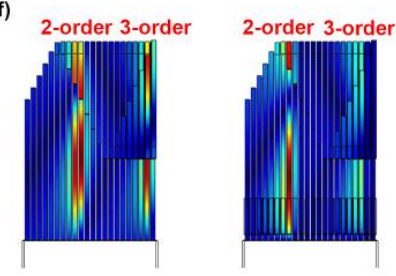

B2: $1580 \mathrm{~Hz}$ $A_{\mathrm{B} 2}=0.90, P_{\mathrm{B} 2}=40.1$

O3: $1980 \mathrm{~Hz}$

C3: $2005 \mathrm{~Hz}$ $A_{\mathrm{c} 3}=0.79, P_{\mathrm{C} 3}=19.6$

B3: $2395 \mathrm{~Hz}$ $A_{\mathrm{B} 3}=0.95, P_{\mathrm{B} 3}=22.9$

(g)
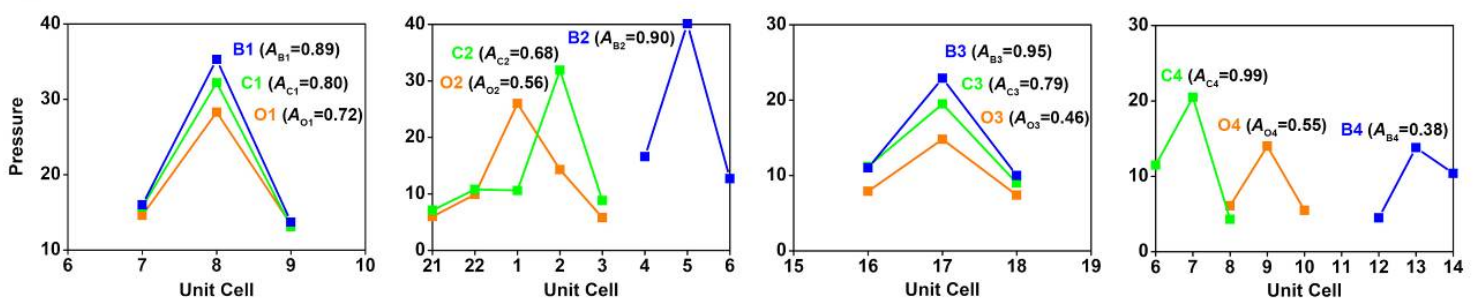

FIG. 3. (a) 1-order, 2-order and 3-order modes within the range of $500 \mathrm{~Hz}$ to $3000 \mathrm{~Hz}$ for $\mathrm{O} / \mathrm{B} / \mathrm{C}$ cases. (b) The absorption coefficient within the range of $500 \mathrm{~Hz}$ to $3000 \mathrm{~Hz}$ for the metasurfaces without bridge $(\mathrm{O}$, orange), with cavity $(\mathrm{C}$, green) and with bridge ( $\mathrm{B}$, blue). The average absorption coefficient within $600 \mathrm{~Hz}$ to $2600 \mathrm{~Hz}$ is $0.56,0.78$, and 0.90 for the cases of $\mathrm{O}$, C and $\mathrm{B}$, respectively. (c-f) The acoustic pressure amplitude field distributions in the supercells for O1-O4, C1-C4, B1-B4, respectively. (g) The absorption coefficient, sound pressure amplitude in the resonant channels, and sound pressure amplitude in their adjacent channels for all interested modes. 
Based on the above theoretical and numerical results and analysis, we have obtained a summary for original straight channel and the $\mathrm{O} / \mathrm{C} / \mathrm{B}$ cases as shown in Table 1 . We list the expressions or calculated values of real filling ratio, effective filling ratio, ideal absorption, simulated averaged absorption, and FP resonant frequency for different cases.

In the following, we discuss the loss effect in the proposed metastructure. Ignoring the mutual impedances in Eq. (13) and observing Eq. (1), the loss in a single channel is decided by the dissipation coefficients $\beta$ and the effective filling ratio $\gamma$. The parameter of $\beta$ is related to the channel thickness $\left(t_{1}=0.15 \mathrm{~cm}\right)$ and the thermal viscous boundary layer thickness [24], expressed as:

$$
d_{\mathrm{visc}}=0.22 \mathrm{~mm} \times \sqrt{100 / f_{n}}
$$

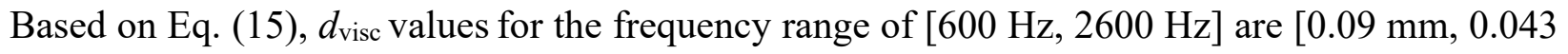
$\mathrm{mm}$, which is about $1 / 17$ to $1 / 35$ of the channel thickness. The dissipation coefficient $\beta$ is related to this size comparison, and can be usually extracted from the experimental results $[2,20]$. Also, the sound absorption at FP resonance [21] is much larger than the non-resonant case, which means FP resonance has a sufficiently ideal $\beta$ for high absorption. Therefore, the absorption coefficient for a single channel is mainly decided by the effective filling rate $\gamma, v i z$, the acoustic impedance matching ability between incident wave and the inner channels. In the Table, the effective filling ratios for $\mathrm{C}$ and $\mathrm{B}$ cases are larger than their real filling ratios. The ideal average absorption is calculated by Eq. (5) with $A=4 \gamma /(1+\gamma)^{2}$, whose results agree well the simulated average absorption in the Table, which validate above analysis for a single channel. The final absorption curves are also related to FP resonant frequencies distributions and the coupling effects from mutual impedances. 
TABLE. The results for cases of Straight Channel (S), Without Bridge (O), With Cavity (O), and With Bridge (B)

\begin{tabular}{ccccc}
\hline & $\mathrm{S}$ & $\mathrm{O}$ & $\mathrm{C}$ & $\mathrm{B}$ \\
\hline Real filling ratio & $\gamma_{0}=0.7$ & $\gamma_{1}=0.2$ & $\gamma_{1}=0.2$ & $\gamma_{1}=0.2$ \\
Effective filling ratio & $\gamma_{0}=0.7$ & $\gamma_{\mathrm{O}}=0.2$ & $\gamma_{\mathrm{C}}=0.41$ & $\gamma_{\mathrm{B}}=0.61$ \\
$\begin{array}{c}\text { Ideal Absorption } \\
\text { Simulated }\end{array}$ & $A_{\text {ideal0 } 0=0.97}$ & $A_{\text {idealO }}=0.56$ & $A_{\text {idealC }}=0.82$ & $A_{\text {idealD }}=0.94$ \\
Absorption & $A_{0}=0.92$ & $A_{\mathrm{O}}=0.56$ & $A_{\mathrm{C}}=0.79$ & $A_{\mathrm{B}}=0.90$ \\
FP Resonant Freq. & $\frac{(2 m-1) c_{0}}{4 L(n)}$ & $\frac{(2 m-1) c_{0}}{4 L(n)}$ & $\frac{(2 m-1) c_{0}}{4 L(n)-h_{1}}$ & $\frac{(2 m-1) c_{0}}{4 L(n)-4 h(n)}$ \\
\hline
\end{tabular}

Then, we have also investigated the sound absorption for oblique incidence case, to study the effect of the incident angle on the absorption. Figure 4(a) shows the absorption for the O/C/B cases with $0,30,45$, and 60 degree incidences in $x$-z plane. Figure 4(b) exhibits the absorption for the $\mathrm{O} / \mathrm{C} / \mathrm{B}$ cases with $0,30,45$, and 60 degree incidences in $y$-z plane. These simulated results suggest that the absorption decreases slightly with large incident angles. This phenomenon is normal because the FP resonance and the surface acoustic impedance are related to incident direction [27]. 
(a)
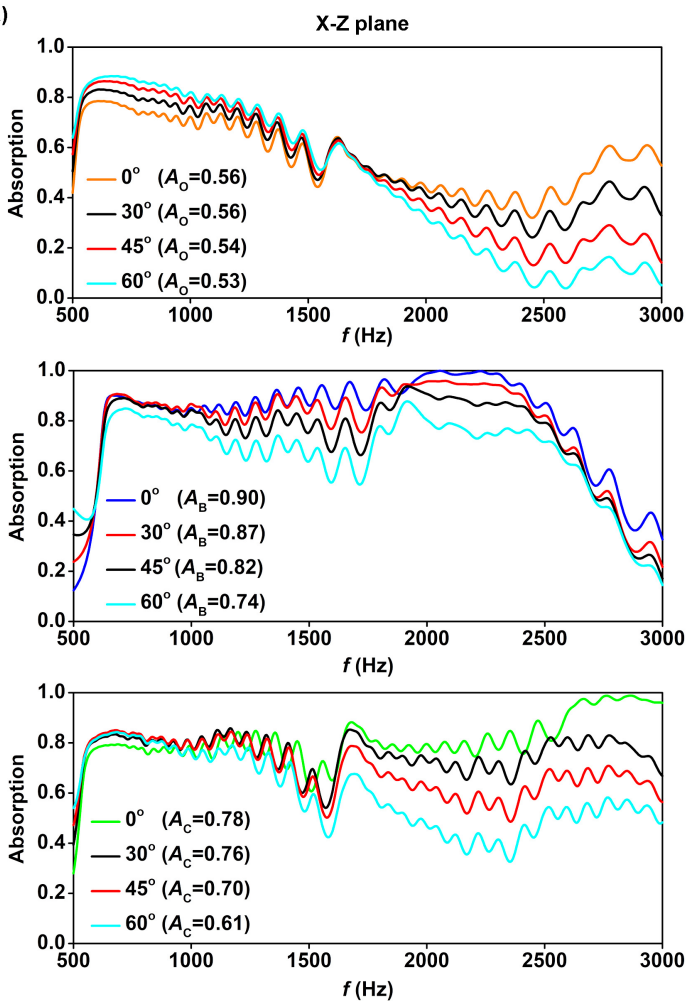

(b)
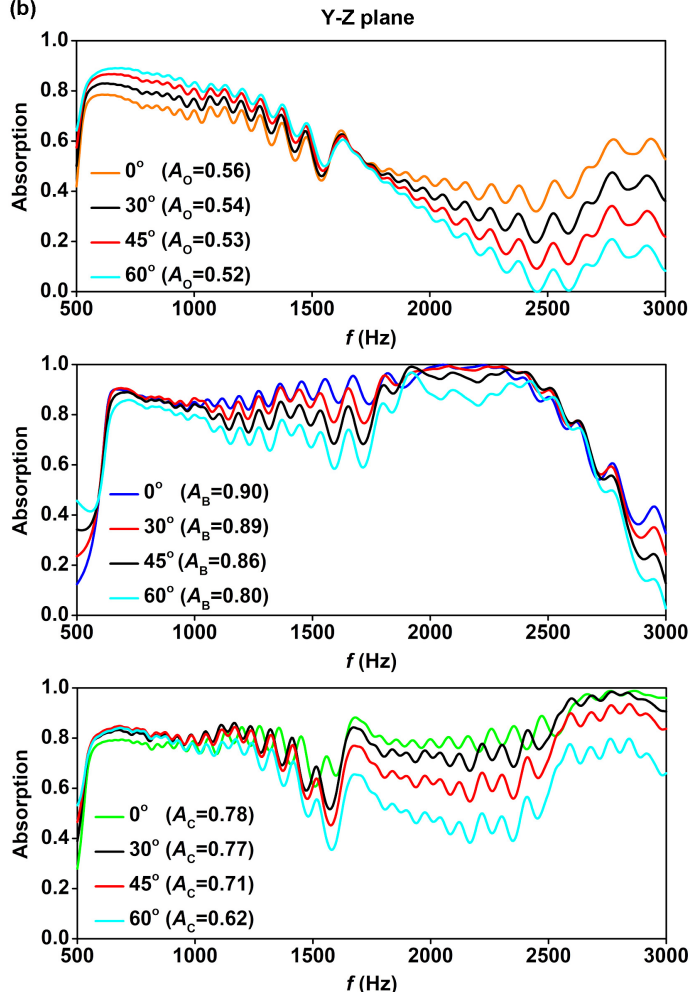

FIG. 4. (a) The simulated absorption for the $\mathrm{O} / \mathrm{C} / \mathrm{B}$ cases with $0,30,45$, and 60 degree incidences in $x-z$ plane. (b) The simulated absorption for the O/C/B cases with $0,30,45$, and 60 degree incidences in $y-z$ plane.

\section{Experimental evidence}

Finally, we show here the experimental demonstration of the proposed models of the $\mathrm{O} / \mathrm{B} / \mathrm{C}$ cases, respectively. Three samples have been fabricated by 3D printing as shown in Fig. 5(a) with the same size of $4.8 \mathrm{~cm} \times 4.8 \mathrm{~cm} \times 6.8 \mathrm{~cm}$ (considering the wall thickness). They are made of polylactic acid (PLA) material with Young's modulus of $E_{P L A}=3.2 \times 10^{9} \mathrm{~Pa}$, mass density of $\rho_{P L A}=1250 \mathrm{~kg} / \mathrm{m}^{3}$, and Poisson's ratio of $v_{P L A}=0.35$. The experimental setup is shown in Fig. 5(b), illustrating a lab-made acoustic impedance tube with a size of $5 \mathrm{~cm} \times 5 \mathrm{~cm} \times 22 \mathrm{~cm}$ to measure the absorption spectrum. The simulated and experimental results are shown in Fig. 5(c). The simulated 
average absorptions within the frequency of $[600 \mathrm{~Hz}, 2600 \mathrm{~Hz}]$ for the $\mathrm{O} / \mathrm{C} / \mathrm{B}$ cases are $0.56,0.78$, and 0.90 , respectively. The corresponding measured average absorptions are $0.65,0.81$ and 0.94 , respectively. The measured results are slightly higher than the simulated ones due to the imperfect sound insulation of the sample at the back wall.

The metastructure for the B case is the desired NAMA design, whose simulated absorption is larger than 0.8 within $[640 \mathrm{~Hz}, 2554 \mathrm{~Hz}]$ frequency range, and the measured absorption is larger than 0.8 within $[640 \mathrm{~Hz}, 2828 \mathrm{~Hz}]$. The numerical and experimental bandwidths $(A>0.8)$ are both larger than 2 octaves. These results suggest that the bandwidth of the NAMA is wider than the previous designs [11-12], and the absorption curve is plain enough, showing the advantage of the nonlocal effect for low-frequency acoustic absorption.

(a)

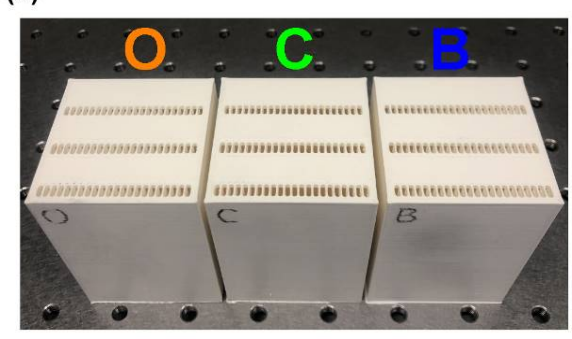

(b)

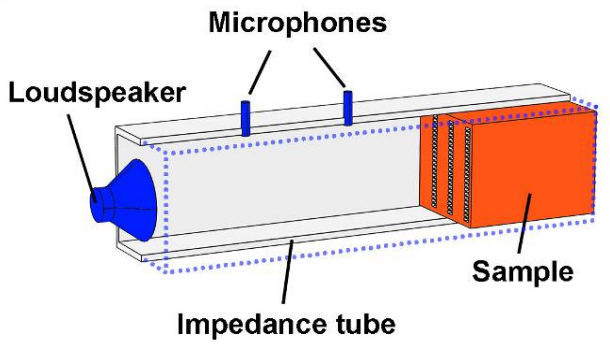

(c)

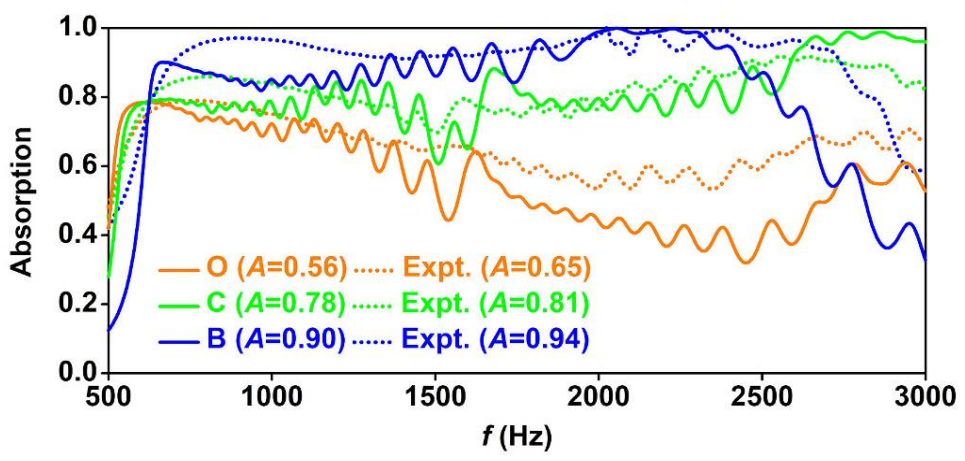

FIG. 5. (a) The photograph of the 3D printed samples for $\mathrm{O} / \mathrm{C} / \mathrm{B}$ cases with a same size of 4.8 $\mathrm{cm} \times 4.8 \mathrm{~cm} \times 6.8 \mathrm{~cm}$. (b) The experimental setup. (c) The simulated and experimental absorption 
for the $\mathrm{O} / \mathrm{C} / \mathrm{B}$ cases within $500 \mathrm{~Hz}$ to $3000 \mathrm{~Hz}$.

\section{Conclusion}

We have conceived and experimentally demonstrated a nonlocal acoustic metasurface absorber showing high broadband absorption coefficient with a very low surface filling ratio. This breaks the limitation of conventional quarter-wavelength resonators based meta-absorber. Theoretical models have been developed to theoretically analyze the proposed meta-structures. By comparing the proposed nonlocal metasurface (B case) with two traditional metasurfaces $(\mathrm{O}$ and C cases), we show that the nonlocality can introduce three specific effects, that is, the optimization of the effective acoustic impedances (characterized as effective filling ratio), the shift of the FabryPerot resonant frequencies, and the enhancement of the coupling effects between adjacent unit cells. This can improve the efficiency and the bandwidth of sound absorption. We have evidenced an ultra-broadband absorption of NAMA over 2 octaves with $A>0.8$. The average absorption is about 0.9 within $[600 \mathrm{~Hz}, 2600 \mathrm{~Hz}$ ] frequency range. The nonlocal acoustic metasurface is subwavelength with a thickness of $\lambda / 9$. The proposed strategy can facilitate the design of acoustic absorbers based on multiple resonant units with improved bandwidth and flexible design, which could have practical applications in noise control and acoustic sink.

\section{Acknowledgments}

This work is supported by the Air Force Office of Scientific Research under award number FA9550-18-1-7021, and by la Région Grand Est and Institut Carnot ICEEL.

\section{Reference}


[1] B. Assouar, B. Liang, Y. Wu, Y. Li, J. C. Cheng, and Y. Jing, Nat. Rev. Mater. 3, 460 (2018).

[2] G. C. Ma, M. Yang, S. W. Xiao, Z. Y. Yang, and P. Sheng, Nat. Mater. 13, 873-878 (2014).

[3] Y. Li and B. Assouar, Appl. Phys. Lett. 108, 063502 (2016).

[4] J. F. Li, W. Q. Wang, Y. B. Xie, B. I. Popa, and S. A. Cummer, Appl. Phys. Lett. 109, 091908 (2016).

[5] K. Donda, Y. F. Zhu, S. W. Fan, L. Y. Cao, Y. Li, and B. Assouar, Appl. Phys. Lett. 115, 173506 (2019).

[6] S. B. Huang, X. S. Fang, X. Wang, B. Assouar, Q. Cheng, and Y. Li, Appl. Phys. Lett. 113, 233501 (2018).

[7] M. Yang, S. Y. Chen, C. X. Fu, and P. Sheng, Mater. Horiz. 4, 673 (2017).

[8] H. Ge, M. Yang, C. Ma, M.-H. Lu, Y.-F. Chen, N. Fang, and P. Sheng, Natl. Sci. Rev. 5, 159 (2018).

[9] N. Jiménez, V. Romero-García, V. Pagneux, and G. Jean-Philippe, Sci. Rep. 7, 13595 (2017).

[10] X. Y. Peng, J. Ji, and Y. Jing, J. Acoust. Soc. Am. 144, EL255-EL261(2018).

[11] Y. F. Zhu, K. Donda, S. W. Fan, L. Y. Cao, and B. Assouar, Appl. Phys. Exp. 12, 114002 (2019).

[12] S. B. Huang, Z. L. Zhou, D. T. Lia, T. Liu, X. Wang, J. Zhu, and Y. Li, Sci. Bull. 65, 373 (2020).

[13] A. C. Eringen, Nonlocal Continuum Field Theories, Springer-Verlag New York (2002).

[14] H. Kwon, D. Sounas, A. Cordaro, A. Polman, and A. Alù, Phys. Rev. Lett. 121, 173004 (2018).

[15] F. Monticone, C. A. Valagiannopoulos, and A. Alù, Phys. Rev. X 6, 041018 (2016).

[16] L. Quan, and A. Alù, Phys. Rev. Appl. 11, 054077 (2019).

[17] Z. L. Hou, X. S. Fang, Y. Li, and B. Assouar, Phys. Rev. Appl. 12, 034021 (2019).

[18] H. Q. Ni, X. S. Fang, Z. L. Hou, Y. Li, and B. Assouar, Phys. Rev. B 100, 104104 (2019).

[19] L. Quan, and A. Alù, Phys. Rev. Lett. 123, 244303 (2019).

[20] M. Yang, G. Ma, Y. Wu, Z. Yang and P. Sheng, Phys. Rev. B 89, 064309 (2014).

[21] X. Jiang, B. Liang, R. Q. Li, X. Y. Zou, L. L. Yin, and J. C. Cheng, Appl. Phys. Lett. 105, 243505 (2014).

[22] L. Kinsler, Fundamentals of Acoustics (Wiley, New York, 1982). 
[23] T. A. Johansson, M. J. Kleiner J. Acoust. Soc. Am. 34, 110, 1315-1328 (2001).

[24] Y. F. Zhu, and B. Assouar, Phys. Rev. B 99, 174109 (2019).

[25] J. Zhu, Y. Y. Chen, X. F. Zhu, F. J. Garcia-Vidal, X. B. Yin, W. L. Zhang, and X. Zhang, Sci. Rep. 3, 1728 (2013).

[26] L. Cao, Z. Yang, Y. Xu, S. W. Fan, Y. Zhu, Z. Chen, Y. Li, and B. Assouar, J. Mech. Phys. Solids 143, 104052 (2020).

[27] Y. X. Gao, Y. P. Lin, Y. F. Zhu, B. Liang, J. Yang, J. Yang, and J. C. Cheng, Sci. Rep. 10(1) 1-6 (2020). 